\title{
Optimal duration of postoperative helmet therapy following endoscopic strip craniectomy for sagittal craniosynostosis
}

\author{
Rajiv R. Iyer, MD, ${ }^{1}$ Xiaobu Ye, MD, MS, ${ }^{1}$ Qiuyu Jin, MD, ${ }^{1}$ Yao Lu, MS, ${ }^{2}$ Luckmini Liyanage, BA, ${ }^{1}$ and \\ Edward S. Ahn, MD'
}

1Division of Pediatric Neurosurgery, Johns Hopkins University School of Medicine, Baltimore, Maryland; and 2Division of Biostatistics and Epidemiology, Weill Cornell Medical College, New York, New York

OBJECTIVE Many infants with sagittal craniosynostosis undergo effective surgical correction with endoscopic strip craniectomy (ESC) and postoperative helmet therapy (PHT). While PHT is essential to achieving optimal cosmesis following ESC, there has been little comprehensive analysis of the ideal PHT duration needed to attain this goal.

METHODS The authors retrospectively reviewed the charts of infants undergoing ESC and PHT for sagittal synostosis at our institution between 2008 and 2015. Data collected included age at surgery, follow-up duration, and PHT duration. Cephalic index $(\mathrm{Cl})$ was evaluated preoperatively $\left(\mathrm{Cl}_{\text {pre }}\right)$, at its peak level $\left(\mathrm{Cl}_{\text {max }}\right)$, at termination of helmet therapy $\left(\mathrm{Cl}_{\text {off }}\right)$, and at last follow-up $\left(\mathrm{Cl}_{\text {final }}\right)$. A multivariate regression analysis was performed to determine factors influencing $\mathrm{Cl}_{\text {final }}$. RESULTS Thirty-one patients ( 27 male, 4 female) were treated in the studied time period. The median age at surgery was 2.7 months (range 1.6 to 3.2) and the median duration of PHT was 10.4 months (range 8.4 to 14.4). The mean $\mathrm{Cl}_{\max }$ was 0.83 (SD 0.01), which was attained an average of 8.4 months (SD 1.2) following PHT initiation. At last follow-up, there was an average retraction of $\mathrm{Cl}_{\text {final }}$ among all patients to 0.78 (SD 0.01). Longer helmet duration after achieving $\mathrm{Cl}_{\max }$ did not correlate with higher $\mathrm{Cl}_{\text {final }}$ values. While $\mathrm{Cl}_{\max }$ was a significant predictor of $\mathrm{Cl}_{\text {final, }}$, neither age at surgery nor $\mathrm{Cl}_{\mathrm{pre}}$ were found to be predictive of final outcome.

CONCLUSIONS Patients undergoing ESC and PHT for sagittal synostosis reach a peak $\mathrm{Cl}$ around 7 to 9 months after surgery. $\mathrm{PHT}$ beyond $\mathrm{Cl}_{\max }$ does not improve final anthropometric outcomes. $\mathrm{Cl}_{\text {final }}$ is significantly dependent on $\mathrm{Cl}_{\max }$, but not on age, nor $\mathrm{Cl}_{\text {pre }}$. These results imply that helmet removal at $\mathrm{Cl}_{\max }$ may be appropriate for $\mathrm{ESC}$ patients, while helmeting beyond the peak does not change final outcome.

https://thejns.org/doi/abs/10.3171/2018.5.PEDS184

KEYWORDS cephalic index; craniosynostosis; endoscopic; helmet therapy; helmeting; orthosis; metopic index; orthotic molding; regression; strip craniectomy; suturectomy; craniofacial

$\mathrm{C}$ RANIOSYNOSTOSIS is characterized by premature fusion of one or more of the cranial sutures resulting in abnormal head shape. The most common type is sagittal synostosis, which results in scaphocephalic and dolichocephalic skull deformity. Recent emphasis on less invasive surgical techniques for craniosynostosis has led to the adoption of early endoscopic strip craniectomy (ESC) and postoperative helmet therapy (PHT) for synostosis treatment early in infancy, a technique pioneered by Jimenez and colleagues nearly 2 decades ago., ${ }^{3,11} \mathrm{Com}-$ pared with traditional calvarial vault remodeling surgery for craniosynostosis, ESC involves smaller incisions, decreased hospital length of stay, and less blood loss, while serving as an effective technique for head shape correction in the young infant population. ${ }^{3,9,11,14}$

Following ESC, patients are fitted with molding orthotic helmets that promote head shape correction by restricting growth in certain directions and allowing cranial expansion in others, a process driven by underlying brain growth. These helmets are worn for 23 hours per day. Serial clinical follow-up in the helmeting period allows for evaluation of head growth and assessment of the progress

ABBREVIATIONS $\mathrm{Cl}=$ cephalic index; $\mathrm{Cl}_{\text {final }}=\mathrm{Cl}$ at last follow-up; $\mathrm{Cl}_{\max }=$ maximum/peak $\mathrm{Cl}$ achieved; $\mathrm{Cl}_{\text {off }}=\mathrm{Cl}$ at helmet removal; $\mathrm{Cl}_{\text {pre }}=$ preoperative $\mathrm{Cl} ; \mathrm{ESC}=$ endoscopic strip craniectomy; $\mathrm{PHT}=$ postoperative helmet therapy.

SUBMITTED January 4, 2018. ACCEPTED May 22, 2018.

INCLUDE WHEN CITING Published online August 31, 2018; DOI: 10.3171/2018.5.PEDS184. 
of postoperative skull reconstruction. While it is generally agreed that PHT is needed to achieve optimal head shape following ESC, there is a lack of comprehensive analysis regarding the duration of helmeting needed to realize optimal cosmesis. ${ }^{8}$ Understanding this parameter is especially important considering potential PHT-associated concerns such as cost, lost wages for visits, quality of life, and helmet-associated morbidity. In this study, we evaluated quantifiable anthropometric outcomes to investigate the optimal duration of PHT required to achieve head shape correction for sagittal synostosis following ESC.

\section{Methods}

\section{Patient Selection, Surgical Technique, and Data Collection}

Patients undergoing ESC and PHT for nonsyndromic sagittal craniosynostosis at our institution between 2008 and 2015 were identified and their cases were retrospectively studied. Surgery was performed by a single surgeon (E.S.A.). All patients underwent a 3 -cm craniectomy spanning from the anterior fontanelle to the lambdoid suture. Early in the experience, barrel staves were performed, but later, barrel staves were not performed..$^{21}$ Data collected included patient sex, age at surgery, length of follow-up, and PHT duration. This study was conducted under Johns Hopkins Medicine IRB approval.

\section{Anthropometric Measurements and Helmeting}

The primary calculated anthropometric measurement was the cephalic index (CI), which is the ratio between the maximum head width (biparietal diameter) and length. The CI was noted at various time points, including preoperatively $\left(\mathrm{CI}_{\text {pre }}\right)$, when the maximum/peak CI was achieved $\left(\mathrm{CI}_{\max }\right)$, at the time of termination of helmet therapy $\left(\mathrm{CI}_{\text {off }}\right.$; referred to in this paper as "helmet removal"), and at last follow-up $\left(\mathrm{CI}_{\text {final }}\right)$. Measurement of CI was performed by physical examination with calipers every 3 months during helmet therapy and for 1 year following helmet removal. Afterward, measurements continued, but were obtained on a less frequent basis. In addition, laser scans were performed at a single helmeting center preoperatively, as well as 1,4 , and $6-8$ weeks postoperatively and 3, 4.5, and 6.5 months postoperatively (Fig. 1). All helmeting was conducted with a single orthotic supplier and single helmet type, the STARlight Bivalve model (Orthomerica Products Inc.). Helmets were generally replaced when the previously manufactured helmet was outgrown, which occurred in approximately 3-month intervals.

\section{Helmet Removal}

The decision to remove the helmet for each patient was made by the senior investigator (E.S.A.). During the study period, the final target CI was 0.8 . In general, helmets were designed to overcorrect the shape as early as possible to allow for an expected retraction in CI after its discontinuation. If the infant's CI was consistently beyond 0.8 at multiple visits separated by 3 months prior to 1 year of age, helmets were generally removed before the first birthday. However, if infants did not reach the 0.8 target, helmet therapy was continued beyond 1 year of age, but typically not beyond 18 months of age.

\section{Statistical Analysis}

Standard descriptive statistics were used to summarize CIs at the defined time points. CIs were compared using the paired Student t-test. A linear regression model was used to calculate the rate of change in CI between time points. Following univariate analysis, a multivariate linear regression model was performed to determine variables
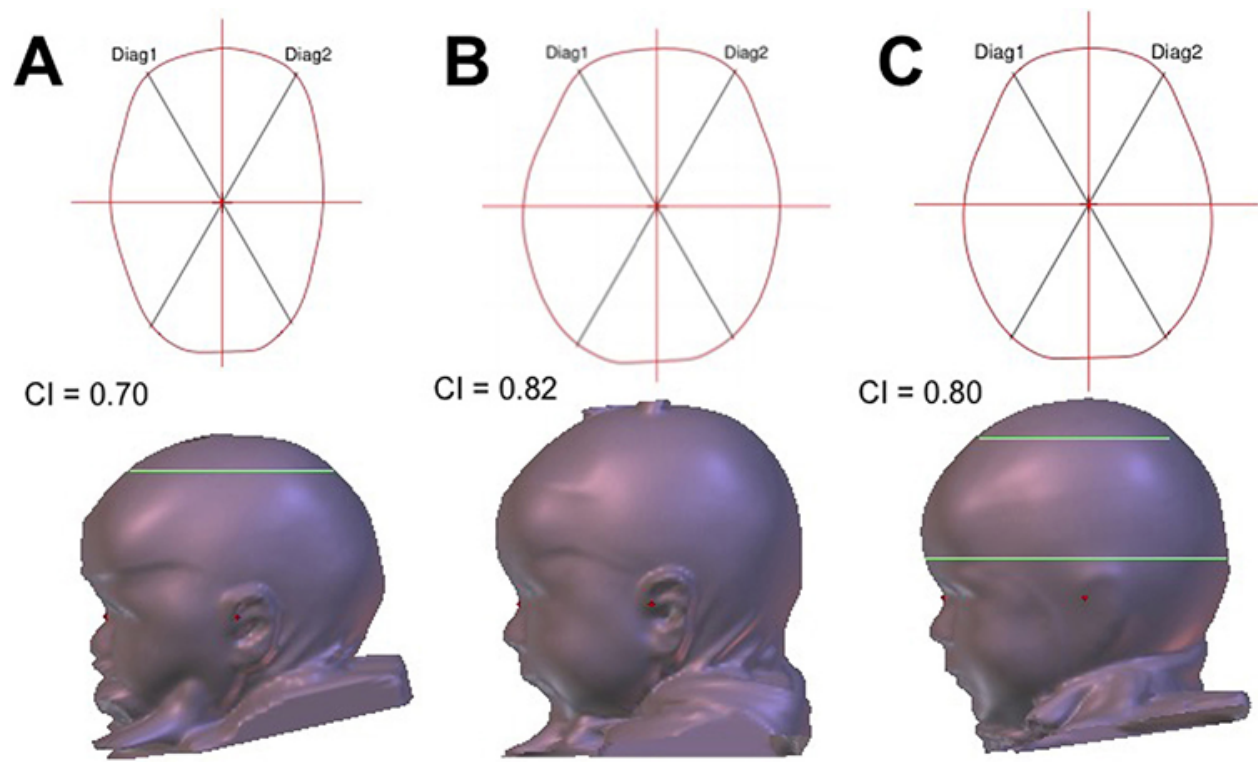

FIG. 1. Serial laser imaging during the post-ESC helmeting period in a patient with sagittal synostosis. Serial lateral 3D laser images (lower row), calculated axial cranial depictions (top row), and corresponding $\mathrm{Cl}$ measurements obtained preoperatively (A), 3 months postoperatively (B), and 12 months postoperatively (C), showing a retraction in $\mathrm{Cl}$ from 0.82 to 0.80 between 3 and 12 months, but an overall improvement in $\mathrm{Cl}$ from 0.70 to 0.80 during the helmeting period. Figure is available in color online only. 
TABLE 1. Cephalic indices following ESC and PHT for sagittal synostosis in 31 patients

\begin{tabular}{|c|c|}
\hline Variable & Value \\
\hline Age at surgery in mos, median (range) & $2.7(1.6-3.2)$ \\
\hline $\mathrm{Cl}_{\text {pre }}$ & $0.68(0.66-0.70)$ \\
\hline Rate $\mathrm{Cl}_{\text {pre }}$ to $\mathrm{Cl}_{\max },{ }^{*}$ mean $\pm \mathrm{SD}$ & $0.02 \pm 0.002$ \\
\hline $\begin{array}{l}\text { Duration of PHT in mos, median } \\
\text { (range) }\end{array}$ & $10.4(8.4-14.4)$ \\
\hline $\mathrm{Cl}_{\max }$ & $0.83(0.82-0.84)$ \\
\hline Time to $\mathrm{Cl}_{\max }$ in mos & $8.4(7.2-9.6)$ \\
\hline $\mathrm{Cl}_{\text {off }}$ & $0.81(0.80-0.83)$ \\
\hline Rate $\mathrm{Cl}_{\text {off }}$ to $\mathrm{Cl}_{\text {final }} \dagger$ & $-0.0024(-0.0036$ to -0.0009$)$ \\
\hline $\mathrm{Cl}_{\text {final }}$ & $0.78(0.77-0.79)$ \\
\hline \multicolumn{2}{|c|}{$\begin{array}{l}\mathrm{Cl}=\text { cephalic index; } \mathrm{Cl}_{\text {final }}=\mathrm{Cl} \text { at last follow-up; } \mathrm{Cl}_{\text {max }}=\text { maximum } / \text { peak } \mathrm{Cl} \mathrm{Cl}_{\text {off }} \\
=\mathrm{Cl} \text { at time of helmet removal; } \mathrm{Cl}_{\text {pre }}=\text { preoperative } \mathrm{Cl} ; \mathrm{PHT}=\text { postoperative } \\
\text { helmet therapy. } \\
\text { Values are the mean }(95 \% \text { confidence interval) unless otherwise indicated. } \\
{ }^{*} \text { Calculated rate of change per month between } \mathrm{Cl}_{\text {pre }} \text { and } \mathrm{Cl}_{\text {max* }} \\
\dagger \text { Calculated rate of change per month in time period between } \mathrm{Cl}_{\text {off }} \text { and } \mathrm{Cl}_{\text {final. }}\end{array}$} \\
\hline
\end{tabular}

associated with $\mathrm{CI}_{\text {final }}$. Significance was defined as a $\mathrm{p}$ value $\leq 0.05$, two-sided. No alpha correction was performed for repeated analyses. Analyses were performed using the SAS software (version 9.3; SAS Institute) or Statistical Packages in $\mathrm{R}$.

\section{Results}

A total of 31 patients (27 male, 4 female) with sagittal synostosis were treated with ESC and PHT in the evaluated time period (Table 1). The patients' median age at surgery was 2.7 months (range 1.6-3.2 months). The me- dian duration of PHT was 10.4 months (range 8.4-14.4 months). Patients were followed for a median of 2.8 years (range 1.0-5.2 years). Helmets were generally replaced every 3 months and the mean number of helmets manufactured and worn per patient was 2.8 (SD 0.47).

The mean $\mathrm{CI}_{\text {pre }}$ was 0.68 (95\% confidence interval 0.66-0.70) and the mean $\mathrm{CI}_{\max }$ was 0.83 (95\% confidence interval 0.82-0.84; $\mathrm{p}<0.0001$ ), which was attained at an average of 8.4 months (95\% confidence interval 7.2-9.6) following ESC and PHT initiation (Fig. 2).

Helmet therapy was terminated in 14 cases prior to, or at achievement of $\mathrm{CI}_{\max }$, and 17 patients had helmets removed after achievement of $\mathrm{CI}_{\max }$ (Table 2). Overall, the calculated rate of change in CI per month between $\mathrm{CI}_{\text {pre }}$ and $\mathrm{CI}_{\max }$ was 0.02 (95\% confidence interval 0.0160.0024). The monthly rate of change in $\mathrm{CI}$ between $\mathrm{CI}_{\text {pre }}$ and $\mathrm{CI}_{\max }$ in patients whose helmets were removed before or at $\mathrm{CI}_{\max }$ was 0.014 (SD 0.004), while in patients whose helmets were removed after reaching $\mathrm{CI}_{\max }$ this rate was 0.025 (SD 0.012; p < 0.01). Patients whose helmets were removed prior to/at $\mathrm{CI}_{\max }$ took an average of 11.0 months (SD 2.0 months) to reach $\mathrm{CI}_{\max }$, while patients whose helmets were removed after $\mathrm{CI}_{\max }$ took an average of 6.1 months (SD 2.2 months) to reach this peak $(\mathrm{p}<0.01)$. The average age at $\mathrm{CI}_{\max }$ was 13.7 months (SD 2.2 months) and 8.6 months (SD 2.2 months) for those with helmets removed before/at $\mathrm{CI}_{\max }$ and those with helmets removed after $\mathrm{CI}_{\max }$, respectively $(\mathrm{p}<0.01)$. Comparison of other interval measurements of rate of change in CI showed no statistically significant differences between these subgroups.

Of the patients whose helmets were removed after $\mathrm{CI}_{\max }$, their helmets were removed at an average of 2.5 months after reaching $\mathrm{CI}_{\max }$. Longer helmet duration after achieving $\mathrm{CI}_{\max }$ did not correlate with higher $\mathrm{CI}_{\text {final }}$ values.

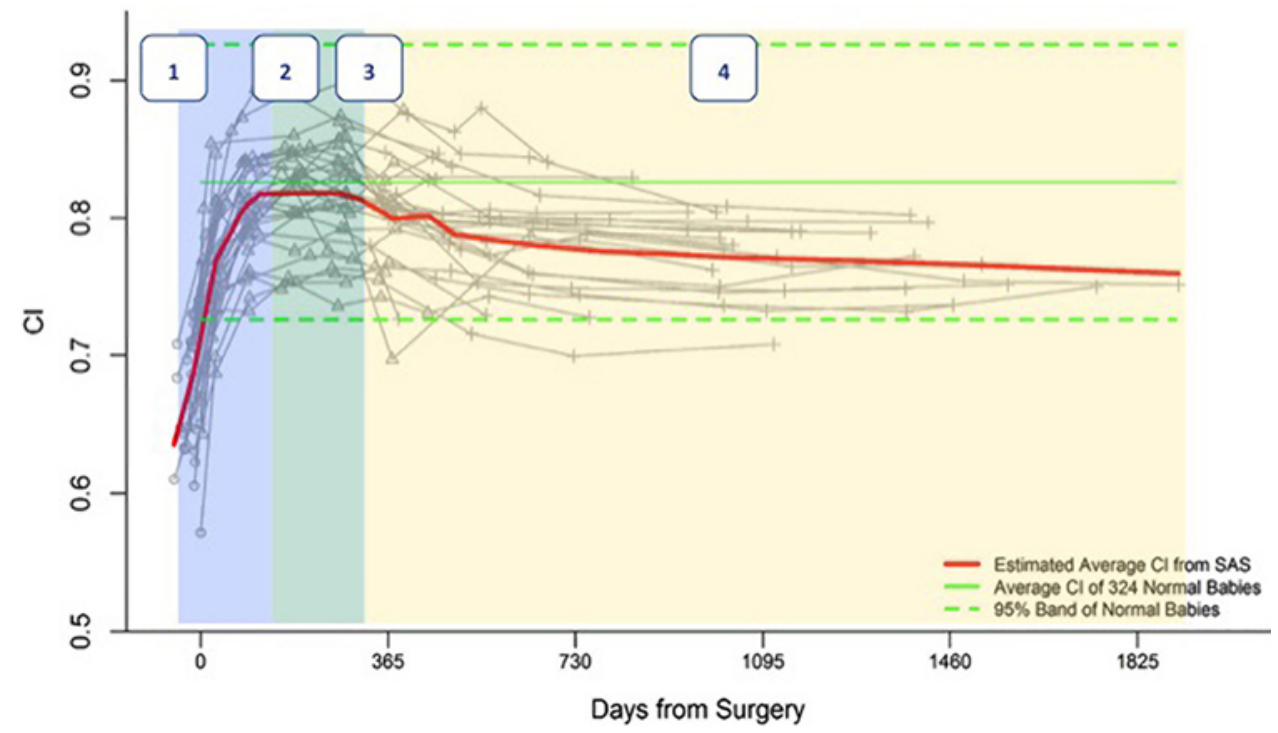

FIG. 2. Cephalic index $(\mathrm{Cl})$ trends in 31 patients undergoing ESC and PHT for sagittal synostosis. The average trend in $\mathrm{Cl}$ in patients treated with PHT after ESC for sagittal synostosis demonstrates a peak around 8 months, followed by a steady retraction to final $\mathrm{Cl}$ measurement, ending in the normal range (red line). The average $\mathrm{Cl}$ in more than 300 normal babies (solid green line) and $95 \%$ normal range (dashed green lines) are shown for comparison. ${ }^{17}$ Numbers listed at the top indicate time points for the average values across groups: $1, \mathrm{Cl}_{\text {pre }} ; 2, \mathrm{Cl}_{\text {max }} ; 3, \mathrm{Cl}_{\text {off }} ;$ and $4, \mathrm{Cl}_{\text {final }}$. Figure is available in color online only. 
TABLE 2. Subgroup $\mathrm{Cl}$ analysis

\begin{tabular}{|c|c|c|c|}
\hline Patient Subgroup & Helmets Removed Before/At CI $I_{\max }(n=14)$ & Helmets Removed After $\mathrm{Cl}_{\max }(\mathrm{n}=17)$ & $\mathrm{p}$ Value \\
\hline Age at surgery (mos) & $2.51 \pm 0.45$ & $2.75 \pm 0.42$ & 0.13 \\
\hline $\mathrm{Cl}_{\text {pre }}$ & $0.71 \pm 0.04$ & $0.72 \pm 0.04$ & 0.89 \\
\hline $\mathrm{Cl}_{\max }$, median (range) & $0.84(0.82-0.86)$ & $0.82(0.80-0.84)$ & 0.12 \\
\hline Rate $\mathrm{Cl}_{\text {pre }}$ to $\mathrm{Cl}_{\max }$ & $0.014 \pm 0.004$ & $0.025 \pm 0.012$ & $<0.01$ \\
\hline Time to $\mathrm{Cl}_{\max }$ (mos) & $11.0 \pm 2.0$ & $6.1 \pm 2.2$ & $<0.01$ \\
\hline Age at $\mathrm{Cl}_{\max }(\mathrm{mos})$ & $13.7 \pm 2.2$ & $8.6 \pm 2.2$ & $<0.01$ \\
\hline Rate $\mathrm{Cl}_{\max }$ to $\mathrm{Cl}_{\text {final }}$ & $-0.002 \pm 0.005$ & $-0.002 \pm 0.002$ & 0.96 \\
\hline Rate $\mathrm{Cl}_{\text {off }}$ to $\mathrm{Cl}_{\text {final }}$ & $-0.003 \pm 0.003$ & $-0.002 \pm 0.004$ & 0.57 \\
\hline $\mathrm{Cl}_{\text {final }}$ & 0.80 & 0.76 & $<0.01$ \\
\hline Age at helmet removal (mos) & $13.3 \pm 1.8$ & $13.3 \pm 1.6$ & 0.95 \\
\hline
\end{tabular}

Values are the mean $\pm S D$ unless otherwise indicated.

The calculated rate of change in $\mathrm{CI}$ per month from $\mathrm{CI}_{\max }$ to $\mathrm{CI}_{\text {off }}$ in this group was 0.006 (95\% confidence interval -0.018 to 0.031 ). At the time of helmet removal, the mean $\mathrm{CI}_{\text {off }}$ was 0.81 (95\% confidence interval $\left.0.80-0.83\right)$.

Ultimately, patients who had their helmets removed after reaching $\mathrm{CI}_{\text {max }}$ had a $\mathrm{CI}_{\text {final }}$ of 0.76 , compared to a $\mathrm{CI}_{\text {final }}$ of 0.80 for those who underwent helmet removal before or at $\mathrm{CI}_{\max }(\mathrm{p}<0.01)$. At last follow-up, there was an average retraction of $\mathrm{CI}_{\text {final }}$ among all patients to 0.78 (95\% confidence interval 0.77-0.79). The calculated rate of change in CI per month in this time period between $\mathrm{CI}_{\text {off }}$ and $\mathrm{CI}_{\text {final }}$ was -0.0024 (95\% confidence interval -0.0036 to $-0.0009)$. There was no statistically significant difference in rate of CI retraction between patients whose helmets were removed before/at $\mathrm{CI}_{\max }$ and patients whose helmets were removed after achieving $\mathrm{CI}_{\max }$.

A univariate regression analysis was performed to determine factors correlating with $\mathrm{CI}_{\text {final }}$ and identified $\mathrm{CI}_{\text {pre }}$, $\mathrm{CI}_{\max }$, and helmet removal after reaching $\mathrm{CI}_{\max }$ as possible variables. Following multivariate regression analysis, $\mathrm{CI}_{\text {pre }}$ was not found to be a significant predictor of $\mathrm{CI}_{\text {final }}(\mathrm{p}=$ 0.73 ), but the absolute value of $\mathrm{CI}_{\max }$ was a significant predictor of $\mathrm{CI}_{\text {final }}(\mathrm{p}<0.0001)$ after adjusting for baseline $\mathrm{CI}$ and helmet removal after $\mathrm{CI}_{\max }$, which had a negative effect on $\mathrm{CI}_{\text {final }}$. Age was not a significant predictive factor of $\mathrm{CI}_{\text {final }}$.

\section{Discussion}

ESC for craniosynostosis involves minimally invasive surgical release of abnormally fused cranial sutures followed by custom cranial molding orthotics that allow for skull reconstruction during the period of rapid underlying brain growth early in infancy. ${ }^{3,11}$ This technique has been implemented by many neurosurgeons instead of traditional calvarial vault remodeling procedures for craniosynostosis due to reduced intraoperative blood loss, hospital length of stay, patient morbidity, and cost. . $^{12,14,18,19}$ While technical variations exist for ESC, such as narrow versus wide vertex craniectomies, barrel-staving versus no barrel-staving, and single-incision versus multipleincision surgery, aesthetic outcomes are similar., ${ }^{6,10,18,21}$ Nonetheless, the evolution of intraoperative techniques for
ESC continues to improve surgical safety, reduce operative times, and minimize patient morbidity.

Although surgical techniques for ESC vary, there is little doubt regarding the role of PHT for achieving desired postoperative head shape and for preventing reversion to the preoperative phenotype due to recurrent suture closure. PHT generally consists of the use of custom-generated molding orthotics that serially correct skull deformity by directing brain growth toward areas requiring cranial expansion. ${ }^{12}$ Early reports of ESC and PHT by Jimenez and Barone nearly 2 decades ago describe the use of cranial molding helmets in 4 patients undergoing ESC, noting that significant changes in head shape occurred even within 1-2 weeks postoperatively with use of this technique. ${ }^{11}$ Another technique for postoperative correction is insertion of a spring cranioplasty device; PHT is avoided, but patients require a separate procedure for device removal. 5,15

Following ESC, helmets are worn approximately 23 hours per day, and as the infant grows, 2 or even 3 serial helmets may be needed to achieve final deformity correction. ${ }^{12}$ While helmets are generally well tolerated, some issues may arise with serial, continuous helmet use over a long duration. For example, superficial wound breakdown or skin infection may occur from continuous scalp pressure, requiring temporary helmet removal, an issue that has been minimized with aerating helmet fenestrations and improved internal cushioning. ${ }^{12,20}$ Additionally, the cost of helmets is on the order of \$800-\$2200, which can be prohibitive for families whose infants require multiple helmets for head shape correction, although cost analyses demonstrate a significant overall cost savings with ESC and PHT when compared to traditional open cranial vault remodeling procedures. ${ }^{1,4,12,16}$ Lost wages for clinic and helmet center visitations should also be factored in, particularly when longer PHT duration is required. Finally, poor helmet compliance is not uncommon, especially toward the end of the PHT period, and can result in treatment failure..$^{20}$

Given the possible issues with PHT that compound with time, a better understanding of the ideal duration of PHT needed to achieve optimal correction is important. In practice, there is some heterogeneity between surgeons in 
the management of helmets, with possible inconsistency even among single surgeons. Some groups report the practice of helmet removal once the desired anthropometric result is obtained or at (or around) 1 year's duration of PHT $^{4,12}$ Evidence of CI retraction after the peak level also raises questions about the durability of various surgical techniques and whether PHT duration plays a role in this phenomenon. . $^{2,4,18}$ These ambiguities have highlighted the need for further investigation of optimal PHT duration following ESC.

In this study, we evaluated anthropometric measurements in sagittal synostosis patients undergoing ESC and PHT to better identify factors affecting cosmetic outcome. Peak CI occurred approximately 8.5 months following ESC and PHT initiation. Continued helmeting after peak CI was reached did not improve final outcomes. In fact, patients who discontinued PHT after reaching their peak CI were found to have significantly worse anthropometrics at last follow-up. This finding is likely attributable to the pattern of PHT continuation for longer periods in patients who were found to have worse appearance and anthropometrics, rather than the implication of longer PHT duration as a negative factor. Regardless, our findings suggest that helmeting beyond peak CI did not improve final anthropometrics.

More detailed analysis of our cohort identified 2 populations of patients: one group featuring a rapid postoperative peak in CI, occurring around 6 months after surgery, and another group demonstrating a more delayed and slightly higher peak CI, occurring around 11 months following ESC. The subgroup of patients with an early CImax represented the same patients who continued to wear helmets longer (after reaching $\mathrm{CI}_{\max }$ ), while the patients with a later peak in CI stopped PHT either before or at the point of $\mathrm{CI}_{\max }$. Because overall PHT duration was similar between these subgroups, these data highlight, for unclear physiological reasons, 2 populations of patients with earlier and later peak anthropometric response following ESC.

Across subgroups, retraction in CI after its peak measurement was observed at last follow-up, consistent with previous reports. ${ }^{2,18}$ Interestingly, whether peak CI was reached early or late, a similar rate of retraction to $\mathrm{CI}_{\text {final }}$ was observed in both of these subgroups of patients. The group of patients with an early peak in CI experienced a longer duration of retraction at a similar rate, which possibly explains why this group of patients had a lower CI at last follow-up. Nonetheless, for all patients, the final CI was still within normal range, which confirms the longterm efficacy of ESC and PHT for head shape correction in this population. One would surmise from these findings that PHT beyond peak CI does not confer a benefit in final outcome after ESC. Ideally, a more rigorous comparison would prospectively examine shorter and longer PHT independent of response, as our study is limited by its retrospective design.

On multivariate analysis, only $\mathrm{CI}_{\max }$ was found to be a significant predictor of $\mathrm{CI}$ at last follow-up, whereas age and preoperative $\mathrm{CI}$ were not significantly associated with $\mathrm{CI}_{\text {final }}$. Jimenez and Barone have suggested that surgical intervention before 3 months of age is associated with improved final outcomes..$^{13}$ Our data suggest that age at inter- vention did not influence final CI. However, it is important to note that our patient population was a selected population skewed toward younger age within infancy. The older infants with milder phenotypes were included, but our population did not include older infants with severely affected head shapes. It is still conceivable that there is a certain cutoff age beyond which ESC with PHT is not effective. Regardless, our data suggest that younger infants, perhaps younger than a certain cutoff age, did not differ in their outcome with respect to their age at surgery.

Based on this study, infants undergoing ESC and PHT for sagittal synostosis should be carefully monitored around the period of CI peak and plateau. Our evidence suggests that $50 \%$ of patients will reach their peak CI before 9 months following ESC for sagittal synostosis. Given these findings, and the retraction that occurs in the time period between peak CI and the CI at last follow-up, it would be reasonable to remove the helmet once maximal $\mathrm{CI}$ is reached. While a higher frequency of clinical visits around the 7- to 9-month postoperative period may help to more precisely identify the time of CI plateau, this is likely overly burdensome on families. Identification of peak CI is limited in its clinical applicability, as it is only discovered in hindsight. However, in scenarios of CI overcorrection or plateauing CI values in a child whose helmet is nearing the end of its useful life, we may elect not to manufacture a new helmet. By limiting PHT to a shorter time period in some patients, quality of life may be improved with helmet freedom. In addition, families can expect decreases in helmet-associated morbidity and cost.

Our study is not without limitations. Our sample size is limited, and further experience, follow-up, and analysis will determine the effects of various predictive factors on long-term anthropometric and clinical outcomes for sagittal synostosis patients. Furthermore, there was some heterogeneity of surgical technique, which could have affected outcomes. Our study was also limited to the experience of a single surgeon, which may add bias, although it adds consistency to the manner in which patients were managed. Nevertheless, while there were general patterns in helmet discontinuation decision-making, there was no single protocol that was followed. Additionally, we recognize that the retrospective nature of our study limits the ability to make robust conclusions regarding PHT following ESC and that a prospective trial would add considerable insight into this area, especially as variance in response to helmeting is possible between patients. For example, a comparison between short and long duration of helmet therapy with defined protocols and quantified helmet compliance could better determine optimal orthotic duration.

\section{Conclusions}

Our findings demonstrate that patients undergoing ESC and PHT for sagittal synostosis can be classified into populations with either an early or a later peak CI, with an overall average peak CI around 7-9 months. The peak is followed by a retraction to a final CI falling within the normal range at last follow-up. Final anthropometric outcomes following ESC and PHT are not significantly dependent on preoperative CI, helmet removal before or 
after peak CI, or patient age. These results imply that helmeting beyond peak CI does not confer any measurable benefit in patients following ESC.

\section{Acknowledgments}

We would like to thank Heather Morgan of the Level 4 Orthotics and Prosthetics helmeting company for her assistance with orthotics and laser images.

\section{References}

1. Abbott MM, Rogers GF, Proctor MR, Busa K, Meara JG: Cost of treating sagittal synostosis in the first year of life. $\mathbf{J}$ Craniofac Surg 23:88-93, 2012

2. Agrawal D, Steinbok P, Cochrane DD: Long-term anthropometric outcomes following surgery for isolated sagittal craniosynostosis. J Neurosurg 105 (5 Suppl):357-360, 2006

3. Barone CM, Jimenez DF: Endoscopic craniectomy for early correction of craniosynostosis. Plast Reconstr Surg 104:1965-1975, 1999

4. Berry-Candelario J, Ridgway EB, Grondin RT, Rogers GF, Proctor MR: Endoscope-assisted strip craniectomy and postoperative helmet therapy for treatment of craniosynostosis. Neurosurg Focus 31(2):E5, 2011

5. Borghi A, Schievano S, Rodriguez Florez N, McNicholas R, Rodgers W, Ponniah A, et al: Assessment of spring cranioplasty biomechanics in sagittal craniosynostosis patients. J Neurosurg Pediatr 20:400-409, 2017

6. Dlouhy BJ, Nguyen DC, Patel KB, Hoben GM, Skolnick GB, Naidoo SD, et al: Endoscope-assisted management of sagittal synostosis: wide vertex suturectomy and barrel stave osteotomies versus narrow vertex suturectomy. J Neurosurg Pediatr 25:674-678, 2016

7. Fearon JA, McLaughlin EB, Kolar JC: Sagittal craniosynostosis: surgical outcomes and long-term growth. Plast Reconstr Surg 117:532-541, 2006

8. Goodrich JT: Single incision endoscope-assisted surgery for sagittal craniosynostosis. Childs Nerv Syst 33:7-8, 2017

9. Han RH, Nguyen DC, Bruck BS, Skolnick GB, Yarbrough CK, Naidoo SD, et al: Characterization of complications associated with open and endoscopic craniosynostosis surgery at a single institution. J Neurosurg Pediatr 17:361-370, 2016

10. Iyer RR, Uribe-Cardenas R, Ahn ES: Single incision endoscope-assisted surgery for sagittal craniosynostosis. Childs Nerv Syst 33:1-5, 2017

11. Jimenez DF, Barone CM: Endoscopic craniectomy for early surgical correction of sagittal craniosynostosis. J Neurosurg 88:77-81, 1998

12. Jimenez DF, Barone CM, Cartwright CC, Baker L: Early management of craniosynostosis using endoscopic-assisted strip craniectomies and cranial orthotic molding therapy.

Pediatrics 110:97-104, 2002
13. Jimenez DF, Barone CM, McGee ME, Cartwright CC, Baker CL: Endoscopy-assisted wide-vertex craniectomy, barrel stave osteotomies, and postoperative helmet molding therapy in the management of sagittal suture craniosynostosis. J Neurosurg 100 (5 Suppl Pediatrics):407-417, 2004

14. Johnson JO, Jimenez DF, Barone CM: Blood loss after endoscopic strip craniectomy for craniosynostosis. J Neurosurg Anesthesiol 12:60, 2000

15. Lauritzen CG, Davis C, Ivarsson A, Sanger C, Hewitt TD: The evolving role of springs in craniofacial surgery: the first 100 clinical cases. Plast Reconstr Surg 121:545-554, 2008

16. Murad GJ, Clayman M, Seagle MB, White S, Perkins LA, Pincus DW: Endoscopic-assisted repair of craniosynostosis. Neurosurg Focus 19(6):E6, 2005

17. Pindrik J, Molenda J, Uribe-Cardenas R, Dorafshar AH, Ahn ES: Normative ranges of anthropometric cranial indices and metopic suture closure during infancy. J Neurosurg Pediatr 25:667-673, 2016

18. Ridgway EB, Berry-Candelario J, Grondin RT, Rogers GF, Proctor MR: The management of sagittal synostosis using endoscopic suturectomy and postoperative helmet therapy. J Neurosurg Pediatr 7:620-626, 2011

19. Vogel TW, Woo AS, Kane AA, Patel KB, Naidoo SD, Smyth MD: A comparison of costs associated with endoscope-assisted craniectomy versus open cranial vault repair for infants with sagittal synostosis. J Neurosurg Pediatr 13:324-331, 2014

20. Wilbrand JF, Wilbrand M, Malik CY, Howaldt HP, Streckbein $\mathrm{P}$, Schaaf $\mathrm{H}$, et al: Complications in helmet therapy. $\mathbf{J}$ Craniomaxillofac Surg 40:341-346, 2012

21. Wood BC, Ahn ES, Wang JY, Oh AK, Keating RF, Rogers $\mathrm{GF}$, et al: Less is more: does the addition of barrel staves improve results in endoscopic strip craniectomy for sagittal craniosynostosis? J Neurosurg Pediatr 20:86-90, 2017

\section{Disclosures}

The authors report no conflict of interest concerning the materials or methods used in this study or the findings specified in this paper.

\section{Author Contributions}

Conception and design: Ahn, Ye. Acquisition of data: Ye, Jin, Lu, Liyanage. Analysis and interpretation of data: Ahn, Iyer, Ye, Jin, Lu. Drafting the article: Iyer, Liyanage. Critically revising the article: Ahn, Iyer. Reviewed submitted version of manuscript: Ahn, Iyer, Ye, Jin. Approved the final version of the manuscript on behalf of all authors: Ahn. Statistical analysis: Ye, Jin, Lu. Study supervision: Ahn.

\section{Correspondence}

Edward S. Ahn: Mayo Clinic, Rochester, MN. ahn.edward@mayo. edu. 